\title{
Pulmonary adiaspiromycosis in a two year old girl
}

\author{
Kyösti Nuorva, Reino Pitkänen, Jouni Issakainen, Niilo-Pekka Huttunen, Matti Juhola
}

\begin{abstract}
A case of disseminated bilateral pulmonary adiaspiromycosis is reportd in a two year old Finnish girl. She recovered from this rare infection after treatment with amphotericin B. She is the first human case of adiaspiromycosis in Scandinavia and she is the youngest child with this disease reported so far. Electron microscopy showed that the three layers of the spore wall were not typical; rather, there seemed to be a gradual transition between the main wall zones, which may be split into an indefinite number of thin layers. Varying numbers and thicknesses were seen with different staining methods, and in different spores. Diagnosis relies on recognition of the fungus in a pulmonary biopsy specimen, because there are no reliable serological tests and culture of the fungus is time consuming and not always successful. It was thought that this patient had become infected as a result of contact with soil dust containing the spores in the yard surrounding her home, and as a result of her mother's work in a large garden shop.

( Clin Pathol 1997;50:82-85)
\end{abstract}

Keywords: adiaspiromycosis; fungal morphology; $\mathrm{Em}$ monsia sp; granulomatous inflammation; electron microscopy.

Adiaspiromycosis is a pulmonary fungal infection of worldwide distribution which affects lower order mammals and, rarely, humans. The causative agents are two variants of the saprophytic soil mould Emmonsia parva (Chrysosporium parvum). Adiaspiromycosis was first discovered in rodents from Transcaucasia (Russia), ${ }^{1}$ and its worldwide distribution in various mammal species was subsequently established. ${ }^{2}$ The first case of adiaspiromycosis in a human was diagnosed in France in 1964, ${ }^{3}$ since when a total of 46 cases of adiaspiromycosis have been reported from France, the Czech Republic, Germany, Spain, Latin America, and the southern United States. ${ }^{4}$ The causative fungi are presently considered to constitute the genus Emmonsia, with a single species $E$ parva. This species is divided into two varieties-namely, $E$ parva var. parva and $E$ parva var. crescens. Recent synonyms include Chrysosporium parvum. ${ }^{15}$ Only $E$ parva var. crescens has been reported to be present in humans. The taxonomy of the Chrysosporiumrelated fungi, which include numerous human pathogens, such as Blastomyces, Histoplasma, and Trichophyton species, may be revised in the light of DNA sequencing. ${ }^{6}$
Both variants produce asexual conidia 2-5 $\mu \mathrm{m}$ in diameter, which, after being inhaled into the lung, are uniquely able to enlarge progressively beyond the ordinary fungal cell size. The variants differ in such a way that the spores of $E$ var. parva do not essentially exceed a diameter of $40 \mu \mathrm{m}$, whereas the $E$ var. crescens spores may grow up to $400 \mu \mathrm{m}$ or more. ${ }^{5}$ It is believed that the conidia do not replicate in the lung, nor do they migrate or disseminate to any extent. ${ }^{7}$ Consequently, adiaspiromycosis is essentially restricted to the lungs. The spherules induce a granulomatous reaction, in which a single spherule is surrounded by a fibronecrotic and suppurative or fibrous granuloma. The disease can be localised and symptomless, or disseminated, occasionally severe, or even fatal. ${ }^{4}$ The severity and extent of the disease in the lungs depends on the amount of the dustborne conidia inhaled.

We present a case of a two year old girl with disseminated pulmonary adiaspiromycosis, who was successfully treated with amphotericin B. She is, as far as we know, the first girl with this disease.

\section{Case report}

The patient, a Caucasian girl, lived in a small town in a new apartment building. The landscaping of the courtyard was under way, with heaps of garden soil around, where the children liked to play. The child's mother was working in a garden shop. The family had no pets. Since the age of one year, the patient had experienced recurrent attacks of wheeze, and at the age of 1.5 years, her respiratory distress was diagnosed as asthma. The treatment consisted of daily inhalations of sodium cromoglycate using a metered dose inhalator with a spacer and additional oral salbutamol during attacks.

At the age of one year and 10 months, the patient became ill with fever and a dry cough. Her breathing was rapid, but without any distress. A general practitioner prescribed oral erythromycin $40 \mathrm{mg} / \mathrm{kg} /$ day. One week later, a chest $x$-ray film revealed diffuse interstitial infiltration in the lungs, and the child was referred to the hospital. On admission, she was well and her temperature was $37.8^{\circ} \mathrm{C}$. She had no dyspnoea and on auscultation her breathing sounds were clear. Her haemoglobin concentration was $104 \mathrm{~g} / 1$ and the peripheral white cell count $18 \times 10^{9} / 1$, with $67 \%$ polymorphonuclear leucocytes, $24 \%$ lymphocytes, and $1.6 \%$ eosinophils. Erythrocyte sedimentation rate (ESR) was $115 \mathrm{~mm} /$ first hour. A Mantoux test yielded negative results. Adenovirus, influenza virus $\mathrm{A}$ and $\mathrm{B}$, cytomegalovirus and parvovirus serologies were negative, nor were any antibodies against Mycoplasma or Legionella sp detected. 

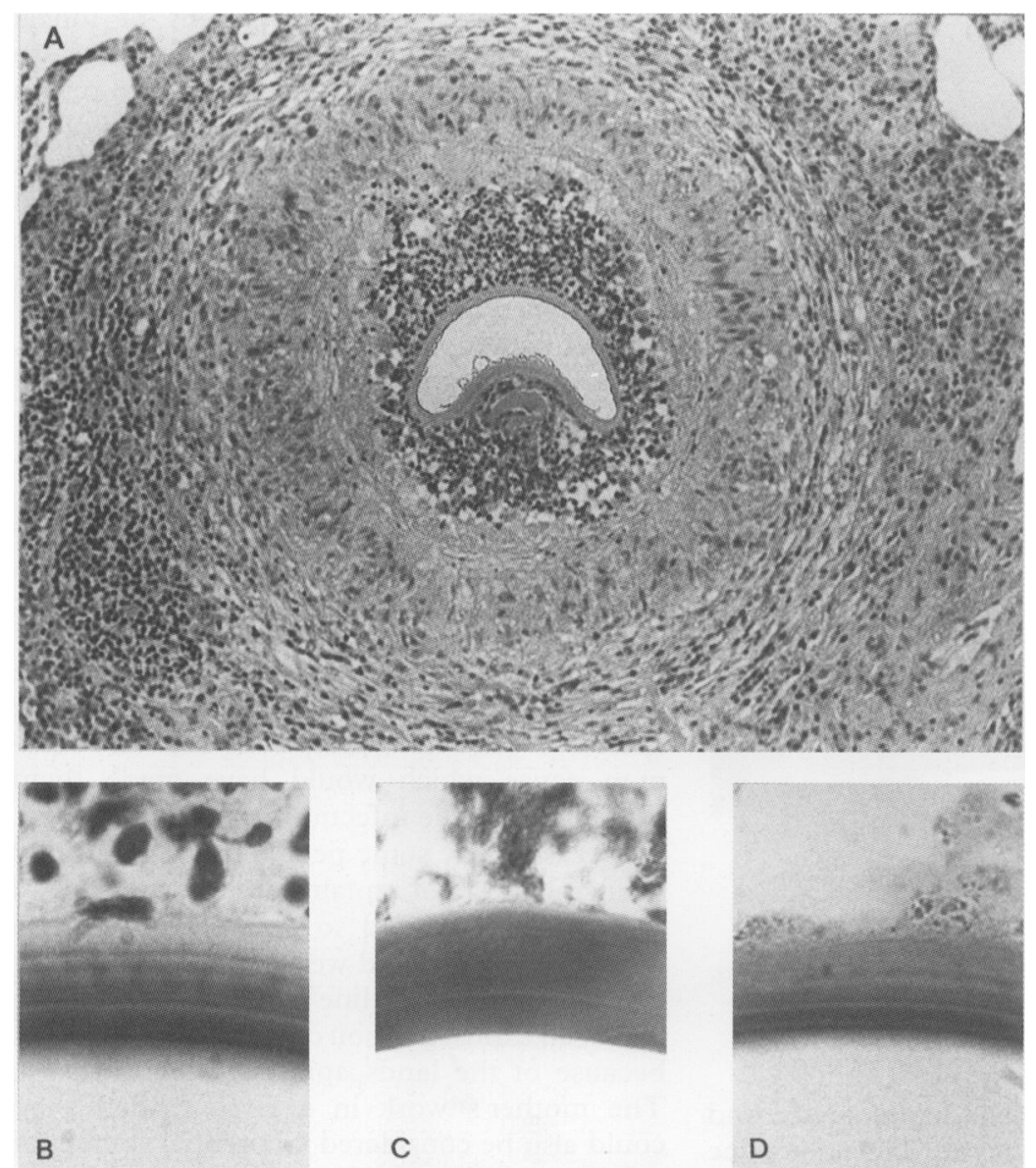

Figure 1 (A) Adiaspore of E parva var. crescens in the centre of a granuloma. The predominant cells from the centre to the periphery are polymorphonuclear and mononuclear leucocytes, epithelioid cells, fibroblasts, and macrophages (haematoxylin and eosin).

Adiaspore wall structure in serial sections visualised by different staining methods: $(B)$ short Grocott-Gomori-methenamine silver; (C) periodic acid Schiff; and (D) Gridley stain.

The chest $x$-ray film showed heavy interstitial infiltration in both lungs. The patient was discharged with a prescription for oral erythromycin.

At follow up two weeks later, she was asymptomatic. The $x$-ray film was unchanged, and she was therefore readmitted for open lung biopsy. Thoracotomy was performed under general anesthesia five weeks after the first symptoms, and a wedge biopsy specimen of the left lower lobe taken. The bacterial culture of the biopsy specimen was negative for Mycobacteria and Legionella sp. The preliminary diagnosis of adiaspiromycosis was made on the basis of histological examination. Sections of the biopsy specimen were specifically kept on mycological media for more than one month, but yielded no growth. In spite of the known presence of the organism, attempts to identify the organism in the tissue by means of phase contrast and fluorescence microscopy with a nonspecific whitening agent (Calcofluor) remained unsuccessful, emphasising the importance of histopathological staining for the diagnosis.

After the diagnosis of pulmonary adiaspiromycosis had been confirmed, a two week regimen of intravenous amphotericin B was started. The $x$-ray film after antifungal treatment had been completed showed a slight decrease in the pulmonary infiltration. One year after the lung biopsy specimen had been taken the patient was clinically well and her chest $x$-ray film was normal. Her asthma treatment continued, but she still wheezes when the pollen count is high.

\section{Histological findings}

A wedge biopsy specimen taken from the left lower lobe of the lung was dilated under negative pressure. The tissue was routinely fixed in neutral formol saline and $5 \mu \mathrm{m}$ sections were stained with haematoxylin and eosin, periodic acid Schiff (PAS), Grocott-Gomori methenamine silver and Gridley stains for light microscopical examiniation.

Microscopically, eight to 25 granulomas were seen in every section (fig 1A). The granulomas were round or ovoid and located subpleurally or interstitially, some of them encroaching the bronchioles or blood vessels. An auramine-rhodamine stain for mycobacteria was negative. About one in four granulomas contained fungal spherules (adiaspores), one in each, but a few of them contained two. Most of the spherules were crescent shaped, obviously due to collapse, but some were round. The measurements were done using a CAS 200 image analyser using the Micrometer 1.0 program (Cell Analysis Systems, Elmhurst, Illinois, USA). The sizes of the granulomas varied from 0.8 to $1.6 \mathrm{~mm}$. The diameters of the spherules varied from 160 to $235 \mu \mathrm{m}$, and the thickness of the spherule wall ranged from 8 to $17 \mu \mathrm{m}$. For each spore, measurements were accepted from only those sections where that individual spore had its widest "equatorial" area, thus eliminating the false positive results caused by tangential sections. Thus the fungus in our case meets the criteria of E. parva var. crescens.

The wall of the spherule was light red on haematoxylin and eosin staining, showing three to five layers. Staining for fungi (PAS, methenamine silver, and Gridley stains) showed that the wall was a complex structure consisting of at least three main layers and several, 12 to 14 , sublayers (figs 1B-1D). The inner main layer, which was 4-7 $\mu \mathrm{m}$ thick, was strongly PAS positive and argyrophilic. In most adiaspores, this layer was homogeneous. The middle main layer was multilaminar (six to eight sublayers) with a thickness of 5-9 $\mu \mathrm{m}$. The outer part of the middle layer showed a porous structure in some spherules on methenamine silver staining. The outer main layer consisted of three to four sublayers. The outermost surface seemed to be partially detached or absent in some spherules. Inside each spherule, a tiny layer or fibres of cytoplasmic material and wide, empty spaces were seen, as a result of storage lipid droplet extraction.

The central parts of the granulomas with spherules were frequently necrotic, containing polymorphonuclear leucocytes and a few lymphocytes. In a few of the spherules, inflammatory cells had invaded the adiaspore. In some granulomas, the number of polymorphonuclear cells was minimal. The middle zone was occupied by epithelioid cells and occasional lymphocytes and macrophages, beyond which there was a concentric layer of fibroblasts 


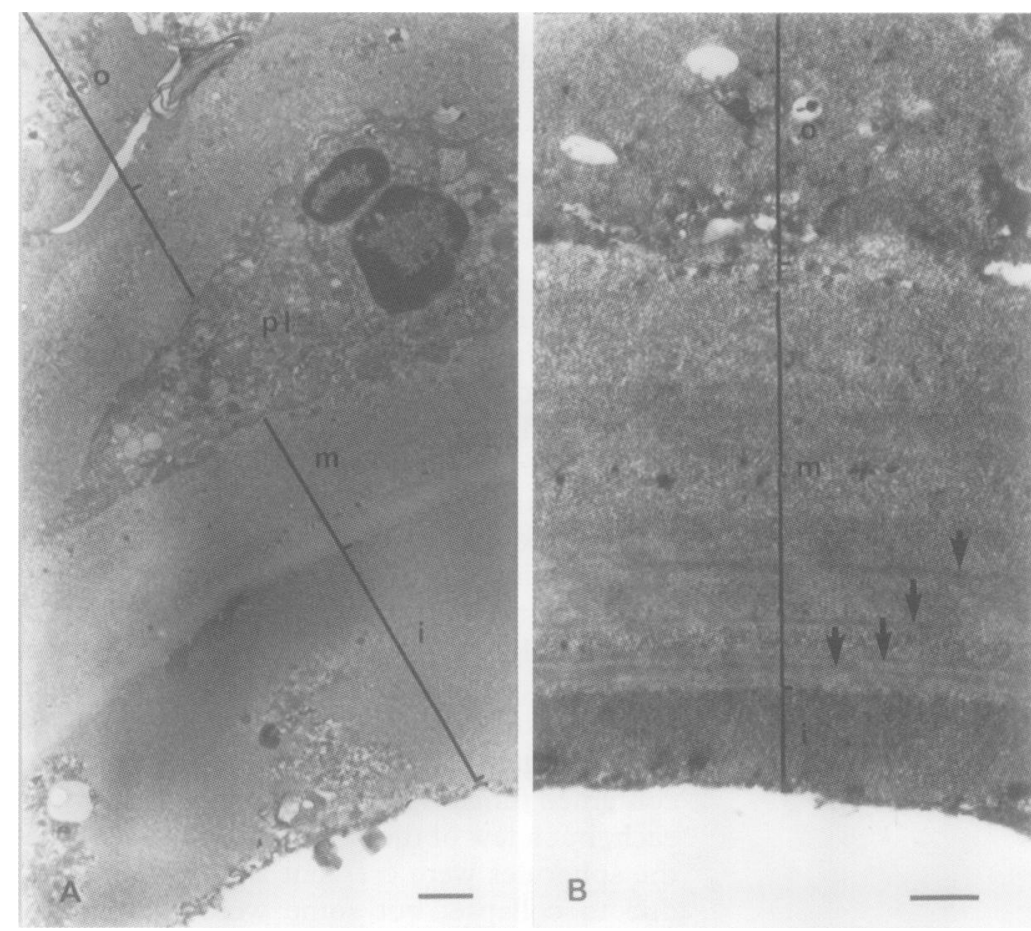

Figure 2 Electron microscopic details of adiaspore wall. Amorphous inner layer (i) with residual cytoplasmic structures, multilaminar middle layer $(m)$ with electron dense granules, and part of the outer layer (o) with amorphous, granular, and vesicular structures. $(A) A$ polymorphonuclear leucocyte $(p l)$ has invaded the middle layer of the adiaspore wall. $(B)$ Multilaminar structure in inner part of middle layer is indicated by arrows. Bar $=1 \mu \mathrm{m}$.

intermixed with plasma cells, lymphocytes, and polymorphonuclear leucocytes. The outer zone was dominated by proliferating macrophages. In the middle and outer zones, a few histiocytic giant cells and PAS positive phagocytes were found.

Electron microscopy verified the complex structure of the adiaspore wall (fig 2). The inner and outer layers were more amorphous than the middle layer. The inner layer was of varying thickness, showing cytoplasmic remnants, including membraneous and vesicular components and homogeneous or slightly fibrillar material. The middle layer, 4-7 $\mu \mathrm{m}$ thick, comprised five to six concentric laminar layers. The outermost main layer consisted of more heterogeneous material and $50-800 \mathrm{~nm}$ wide electron dense inclusions.

\section{Discussion}

Adiaspiromycosis is a fungal disease of the lung caused by two variants of Emmonsia parva. The fungus affects lower order mammals, mostly rodents, and rarely humans, although the organism is distributed worldwide. ${ }^{7}$ The larger variant, $E$ parva var. crescens, is widespread and relatively abundant in Finland, ${ }^{89}$ but to the best of our knowledge, no human cases of the disease have been diagnosed before in Northern Europe.

The mycelial growth of $E$ parva var. crescens in soil produces conidia $2-5 \mu \mathrm{m}$ in diameter, which are inhaled by small mammals or, occasionally, by people. In the lungs of mammals, the conidia begin to extend their diameter and wall thickness without a definite upper limit, but, for some reason, they do not divide. The natural life cycle of $E$ parva is poorly known, and the cases of adiaspiromycosis may repre- sent only random, malformations of fungal development. There is some evidence to suggest, however, that the spores could return in a viable state to the soil after the death of the host mammal and give rise to a new generation of mycelial growth and normal sized aleuriospores. ${ }^{10}$ The incidence and evolutionary importance of this alternative cycle for the fungus remain speculative.

The patient reported here had asthma, which was treated with daily inhalations of sodium cromoglycate, using a metered dose inhalator with a spacer, and additional oral salbutamol during mild attacks. Corticosteroids were not used. Some of the patients reported before have been healthy, while others have had diverse pulmonary disorders, including emphysema, tuberculosis, aspergillosis, and pulmonary abscesses, and two patients have been immunocompromised. ${ }^{4}$ However, among those who have had adiaspiromycosis, no common cause which would have made them susceptible to the infection has been detected. The reason why some people become infected while most do not remains a mystery. Many of the patients reported so far were engaged in outdoor activities and were therefore at risk of exposure to dust. In line with this, our patient had been exposed to soil dust containing spores because of the landscaping of the courtyard. The mother's work in a large garden shop could also be considered a potential source for variable soil inocula. For all that, considering the worldwide occurrence of $E$ parva var. crescens, the cases of manifest disease or even incidental necropsy findings are astonishingly rare.

The patient was treated for two weeks with intravenous amphotericin B $0.5 \mathrm{mg} / \mathrm{kg} /$ day. There have been reports of the use of monotherapy or combinations of amphotericin B, 5-fluorocytosine, ketoconazole, itraconazole, and systemic corticosteroids in pulmonary adiospiromycosis. A patient with osteomyelitis caused by Chrysosporium has also been treated succesfully with amphotericin B. ${ }^{11}$ However, few patients with disseminated adiaspiromycosis of the lung have recovered without treatment. ${ }^{4}$ Because disseminated adiaspiromycosis has been reported to be fatal ${ }^{4}$ and our patient showed heavy diffuse interstitial infiltration in both lungs, she was treated with amphotericin B. A slight decrease in the pulmonary infiltration was seen at the end of treatment, and after one year the chest $x$-ray film was normal. We consider antifungal treatment logical in such cases, because adiaspores are living organisms which provoke an interstitial granulomatous reaction, with compression of the adjacent blood vessels and bronchioli, thus potentially compromising pulmonary function.

According to our observations, the 'three layers' of the spore wall ${ }^{4}$ should not be considered a consistent feature. For the time being, we would like to use the term 'multilaminar'. We emphasise the gradual transition between all the main wall zones, which may progressively and irregularly split into an indefinite number of thin layers. Of these, the outermost 
and partially detached ones may give a false impression of constituting a defined outermost layer. Varying numbers and thicknesses of layers can be seen with different staining methods and in different spores. Diagnosis of this disease rests on recognition of the fungus in a pulmonary biopsy specimen, because there are as yet no reliable serological tests available and culture of the fungus is time consuming and rarely successful.

1 Kwon-Chung KJ, Bennett JE. Medical mycology. Philadelphia: Lea and Febiger, 1992.

2 Jellison WL, Drouhet E, Segretain G, L'Heritier M, Pette F. Adiaspiromycose (haplomycose) chez les mammiferes sauvages en France. Annales de l'Institute Pasteur Lille 1961; 100:747.

3 Doby-Dubois $M$, Chevrel ML, Doby JM, Louvet $M$. Premier cas humain d'adiaspiromycose par Emmonsia crescens, Emmons et Jellison, 1960. Bulletin de la Societé de Pathologie Exotique et de ses Filiales 1964;57:240-4.
4 England DM, Hochholzer L. Adiaspiromycosis: an unusual fungal infection of the lung. Report of 11 cases. Am $\mathcal{F}$ Surg Pathol 1993;17:876-86.

5 de Hoog GS, Guarro J. Atlas of clinical fungi. Baarn, Delft, The Netherlands: Centraal bureau voor Schimmelcultures Reus, Spain: Universitat Rovira i Virgili, 1995.

6 McGinnis MR, Sigler L, Bowman BH, Masuda M, Wang CJK. Impact of conidiogenesis, teleomorph connections, pleomorphism and molecular genetics on evolving hyphomycete systematics. F Med Vet Mycol 1992;30(Suppl 1):261-9.

7 Schwarz J. Adiaspiromycosis. Pathol Annu 1978;13:41-53.

8 Jellison WL, Helminen M, Vinson JW. Presence of a pulmonary fungus in rodents in Finland. Ann Med Exp Biol Fenn 1960;38:361-6.

9 Taarna J. Observations on the occurrence of Emmonsia crescens Emmons \& Jellison in Finland. Archivum Societalis Zoologicae Botanicae Fennicae Vanamo 1962;16:145-51.

10 Křivanec $K$, Otčenášek $M$. Importance of free living mustelid carnivores in circulation of adiaspiromycosis. Mycopathologia 1977;60:139-44.

11 Stillwell WT, Rubin BD, Axelrod JL. Chrysosporium, a new causative agent in osteomyelitis. Clin Orthop 1984;104: $190-2$. 